Yasunori Naganawa - Kohji Itoh • Michie Shimmoto

Kyoko Takiguchi · Hirofumi Doi • Yuzuru Nishizawa

Takayoshi Kobayashi · Sachiko Kamei • Kiven E. Lukong

Alexey V. Pshezhetsky • Hitoshi Sakuraba

\title{
Molecular and structural studies of Japanese patients with sialidosis type 1
}

\begin{abstract}
To gain insight into the pathogenesis of sialidosis type 1, we performed molecular investigations of two unrelated Japanese patients. Both of them are compound heterozygotes for base substitutions of ${ }^{649} \mathrm{G}$-to-A and ${ }^{727} \mathrm{G}$-to-A, which result in amino acid alterations V217M and G243R, respectively. Using homology modeling, the structure of human lysosomal neuraminidase was constructed and the structural changes caused by these missense mutations were deduced. The predicted change due to V217M was smaller than that caused by G243R, the latter resulting in a drastic, widespread alteration. The overexpressed gene products containing these mutations had the same molecular weight as that of the wild type, although the amounts of the products were moderately decreased. A biochemical study demonstrated that the expressed neuraminidase containing a V217M mutation was partly transported to lysosomes and showed residual enzyme activity, although a G243R mutant was retained in the endoplasmic reticulum/Golgi area and had completely lost the enzyme activity. Considering the data, we surmise
\end{abstract}

Y. Naganawa $\cdot$ K. Itoh $\cdot$ M. Shimmoto $\cdot$ H. Sakuraba $(\varangle)$ Department of Clinical Genetics, The Tokyo Metropolitan Institute of Medical Science, Tokyo Metropolitan Organization for Medical Research, 3-18-22 Honkomagome, Bunkyo-ku, Tokyo 113-8613, Japan

Tel. +81-3-3823-2101, ext. 5364; Fax +81-3-3823-6008

e-mail: sakuraba@rinshoken.or.jp

K. Takiguchi $\cdot$ H. Doi

Fujitsu Laboratories Ltd., Chiba, Japan

Y. Nishizawa

Second Department of Internal Medicine, Nippon Medical School, Tokyo, Japan

T. Kobayashi

Department of Neurology, Faculty of Medicine, Tokyo Medical and Dental University, Tokyo, Japan

S. Kamei · Y. Naganawa

School of Allied Health Science, Faculty of Medicine, Tokyo

Medical and Dental University, Tokyo, Japan

K.E. Lukong · A.V. Pshezhetsky

Service de Génétique Médicale, Départment de Pédiatrie, Hôspital

Sainte-Justine, Université de Montréal, Montréal, Canada that the V217M substitution may be closely associated with the phenotype of sialidosis type 1 with a late onset and moderate clinical course.

Key words Sialidosis $\cdot$ Lysosomal neuraminidase $\cdot$ Homology modeling $\cdot$ Protective protein / cathepsin A

\section{Introduction}

Lysosomal neuraminidase (sialidase, EC 3. 2. 1. 18) is an acid glycosidase that catalyzes the removal of the terminal sialic acid residues of glycoconjugates (Verheijen et al. 1982, 1985, 1987; Hiraiwa et al. 1988; van der Horst et al. 1989). Mammalian lysosomal neuraminidase is associated with protective protein/cathepsin A (PPCA; EC 3. 4. 16. 1) and $\beta$-galactosidase (EC 3.2.1.23), and essentially requires PPCA for its activation (d'Azzo et al. 1982; van der Spoel et al. 1998). Defects in the PPCA gene result in a human metabolic disease, galactosialidosis, associated with secondary deficiencies of lysosomal neuraminidase and $\beta$ galactosidase (reviewed by d'Azzo et al. 1995).

The primary defect of the lysosomal neuraminidase causes another autosomal recessive genetic disorder, sialidosis (reviewed by Thomas et al. 1995). The disease is clinically divided into two groups: late-onset sialidosis type 1 , characterized by macular cherry-red spots and myoclonus, and early-onset sialidosis type 2, distinguished from type 1 by the presence of dysmorphic features besides neurological symptoms and cherry-red spots. The molecular basis of the disease has remained obscure. Recently, human lysosomal neuraminidase cDNA was isolated (Bonten et al. 1996; Pshezhetsky et al. 1997; Milner et al. 1997), and studies for clarifying the pathogenesis of sialidosis were started. So far, six different mutations in the neuraminidase gene have been reported (Bonten et al. 1996; Pshezhetsky et al. 1997). However, little has been elucidated regarding the enzyme proteins caused by the mutant genes.

In this study, we characterized the molecular defects in lysosomal neuraminidase caused by gene mutations, which 
were newly identified in two unrelated Japanese patients with sialidosis type 1 .

\section{Patients and methods}

\section{Patients}

Patient 1. This patient is a 25 -year-old female offspring of nonconsanguineous parents. At the age of 17 years, she developed an ataxic gait, and speech disturbance. There were subsequent episodes of generalized convulsions and visual disturbance. Ophthalmological examination disclosed macular cherry-red spots. Her neurological symptoms rapidly progressed, and she could not stand without support at the age of 18 years, and is bed-ridden at present. Her younger sister exhibited a similar clinical course, although her brother had a milder clinical course (Mitoma et al. 1993). Lysosomal enzyme assay revealed an isolated neuraminidase deficiency, but $\beta$-galactosidase activity was normal in these three siblings (Yamamoto et al. 1995).

Patient 2. This patient is a 42-year-old man. He was born to nonconsanguineous parents. He developed normally after birth until the age of 32 years, when involuntary movements began in the upper extremities. Then, he developed gait disturbance and slurred speech. Physical examination at age 41 years revealed dysarthria, action myoclonus, and hyperactive deep tendon reflexes. Lens opacities and macular cherry-red spots were detected. Neither computed tomography nor magnetic resonance imaging of the head revealed any abnormality. Lysosomal enzyme assay disclosed a neuraminidase deficiency. Other enzyme activities, including those of $\beta$-galactosidase and cathepsin $\mathrm{A}$, were normal. His brother has shown similar clinical manifestations, but detailed data for the brother, including neuraminidase activity, are not available.

\section{Materials}

1,2-Dimyristyloxypropyl-3-dimethyl-hydroxy ethylammonium bromide (DMRIE)-C reagent for transfection was purchased from GIBCO/BRL (Grand Island, NY, USA). We purchased leupeptin from the Peptide Institute (Osaka, Japan); N-benzyloxycarbonyl-L-phenylalanyl-L-leucine (ZPhe-Leu) from Sigma (St. Louis, MO, USA); and 1,4-diazabicyclo-[2,2,2]octane (DABCO) from Wako Pure Chemical Industries (Osaka, Japan). Restriction enzymes were purchased from New England Biolabs (Beverly, MA, USA).

\section{Cell culture}

Human skin fibroblasts derived from healthy Japanese subjects and patients with sialidosis type 1 were maintained in Ham's F-10 medium supplemented with $10 \%$ fetal calf serum (FCS) and antibiotics, as described previously (Sakuraba et al. 1999). For transient expression of the wild- type and mutant lysosomal neuraminidase cDNAs, a fibroblast cell line derived from a galactosialidosis patient (ASVGS-1) (Shimmoto et al. 1993; Gluzman et al. 1981) or COS-1 cells (kindly supplied by Dr. Y. Nabeshima, National Institute of Neuroscience, NCNP, Tokyo, Japan) were cultured in the same medium.

\section{Gene analysis}

Total RNA and genomic DNA were isolated from the cultured fibroblasts, as described previously (Davis et al. 1986). Lysosomal neuraminidase cDNA, including the entire protein coding sequence, was obtained by reverse transcription (RT)-polymerase chain reaction (PCR) (Kawasaki 1990), using an oligo(dT) primer and an RT-PCR kit (Amersham, Buckinghamshire, England), followed by cDNA amplification, using a pair of primers (sense, $5^{\prime}$-AGTCTAGCTGC CAGGGTCGC-3' and antisense, 5'-AGTCCTGAAGGC AGAATACC-3'). The amplified lysosomal neuraminidase cDNA was subcloned into the pGEM-T vector (Promega, Madison, WI, USA). Nucleotide sequencing was performed with a DyeDeoxy Terminator Cycle Sequencing Kit and an automated sequencer (Applied Biosystems, Foster City, CA, USA). As described under "Results", ${ }^{649}$ G-to-A (V217M) and ${ }^{727}$ G-to-A (G243R) substitutions were detected in both the patients. These base substitutions are located in exon 4 of the lysosomal neuraminidase gene. To confirm the presence of these mutations, genomic DNA fragments, including exon 4, were amplified by PCR, using sense ( $5^{\prime}$-TGAGCCCCTAGAGTCTCC-3') and antisense (5'-GGCAGGGAGGGTCAAATG-3') primers. The amplified DNA fragment was analyzed by direct sequencing.

\section{Transient expression of lysosomal neuraminidase cDNA}

An expression vector for the wild-type neuraminidase was prepared by inserting the full-length human lysosomal neuraminidase cDNA into the Not I site of the pCMV expression vector (MacGregor and Caskey 1989), and designating this as pCMV-sialidase, as previously described (Vinogradova et al. 1998). For expression of the mutant lysosomal neuraminidase cDNA, the DNA fragment obtained from the mutant cDNA subclone, containing either ${ }^{649} \mathrm{G}$-to-A (V217M) or ${ }^{727} \mathrm{G}$-to-A (G243R), was double-digested with BstEII and Kpn I, and substituted for the pCMV-sialidase plasmid. The cloned plasmids were designated pCMV-sialidase[V217M] and pCMVsialidase[G243R], respectively.

For enzyme assay and immunoblotting, ASVGS-1 and COS- 1 cells were seeded onto 60 -mm dishes $\left(2 \times 10^{5}\right.$ cells $)$, 1 day before transfection. For immunocytochemistry, COS1 cells were seeded onto two-well chamber slides (Lab-Tek; Nunc, Naperville, IL, USA $)\left(1 \times 10^{5}\right.$ cells $)$. The transfection was performed using DMRIE-C reagent (GIBCO/BRL) according to the manufacturer's protocol. Briefly, a mixture comprising the plasmid DNA ( $5 \mu \mathrm{g})$ and DMRIE-C reagent $(10 \mu \mathrm{l})$ was added to cells cultured in FCS-free Ham's F-10 medium, followed by incubation for $5 \mathrm{~h}$. Then, the culture 
medium was replaced with Ham's F-10 medium containing $10 \%$ FCS, and the cells were cultured for 4 days. As for ASVGS-1 cells for enzyme assay and COS- 1 cells for immunocytochemical analysis, a pCAGGS vector (Miyazaki et al. 1991) containing human wild-type PPCA cDNA (pCAGGS-PP, 5 $\mu \mathrm{g}$ ) (Shimmoto et al. 1993) was cotransfected with the wild-type or mutant lysosomal neuraminidase cDNA.

\section{Enzyme assay}

As mentioned in the "Introduction", a sufficient amount of PPCA is required for the activation of lysosomal neuraminidase, and ASVGS-1 cells co-transfected with PPCA cDNA were used as host cells in this analysis. The transfected ASVGS-1 cells were washed with phosphate-buffered saline (PBS), harvested by scraping, and then suspended in distilled water containing $0.1 \mathrm{mM}$ leupeptin. A homogenate was prepared by pipetting, and then neuraminidase activity was measured immediately, according to a method reported previously (Suzuki et al. 1981). The rest of the homogenate was sonicated and used for other lysosomal enzyme assays and protein determination. Glycosidase activities, including those of neuraminidase, $\beta$-galactosidase, and $\beta$ hexosaminidase, were assayed fluorometrically, using 4methylumbelliferyl glycosides as substrates (Suzuki 1987). Cathepsin A activity was measured at pH 5.6, using Z-PheLeu as a substrate (Itoh et al. 1991). Protein determination was performed with a dye-binding assay kit (Bio-Rad, Richmond, CA, USA), using bovine serum albumin (BSA) as a standard.

Polyacrylamide gel electrophoresis and immunoblotting

After transfection, COS-1 cells were harvested, sonicated in distilled water containing $0.1 \mathrm{mM}$ leupeptin, and then centrifuged at $10,000 \times g$ for $15 \mathrm{~min}$ at $4^{\circ} \mathrm{C}$. Aliquots of the resultant supernatant, after reduction with $25 \mathrm{mM}$ mercaptoethanol, were subjected to sodium dodecylsulfatepolyacrylamide gel electrophoresis (SDS-PAGE), according to the method of Laemmli (1970), on a 10\% acrylamide gel (Realgel Plate; Biocraft, Tokyo, Japan). Proteins were visualized by immunostaining, using an anti-neuraminidase antibody (Vinogradova et al. 1998) and a Super Signal Ultra Chemiluminescence Kit (Pierce, Rockford, IL, USA). Prestained SDS-PAGE standards (Bio-Rad, Hercules, CA, USA) were used as molecular mass standard proteins: phosphorylase b, $110 \mathrm{kDa}$; BSA, $84 \mathrm{kDa}$; ovalbumin, $50 \mathrm{kDa}$; carbonic anhydrase, $33 \mathrm{kDa}$; soybean trypsin inhibitor, $24 \mathrm{kDa}$; and lysozyme, $16 \mathrm{kDa}$.

\section{Immunocytochemical analysis}

The transfected COS- 1 cells were fixed with ice-cold $4 \%$ paraformaldehyde/PBS (v/v) and then with $80 \%$ methanol/ PBS (v/v). After nonspecific binding was blocked with 5\% goat serum and $1 \%$ BSA in PBS, the cells were treated with the anti-neuraminidase antibody (Vinogradova et al. 1998) overnight at $4^{\circ} \mathrm{C}$. Then the cells were washed with PBS, followed by treatment with fluorescein-isothiocyanate (FITC)-labeled $\mathrm{F}\left(\mathrm{ab}^{\prime}\right)_{2}$ fragments of goat anti-rabbit IgG (BioSource, Camarillo, CA, USA). After being washed with PBS, the cells were mounted with $55 \%$ glycerol/PBS $(\mathrm{v} / \mathrm{v})$ containing $3.8 \%$ DABCO. The stained cells were examined under a fluorescence microscope (Axiophot; Zeiss, Oberkochen, Germany).

Structural modeling of human wild-type and mutant lysosomal neuraminidases

The crystal structure of neuraminidase from Salmonella typhimurium LT2 was determined (Crennell et al. 1993) and deposited in the Protein Data Bank (PDB; Brookhaven National Laboratory, Upton, NY, USA) (Sussman et al. 1998). On the basis of this information (PDB file: 2sil), models of the human wild-type lysosomal neuraminidase and its mutants were built, using molecular modeling software (SYBYL/COMPOSER; TRIPOS, St. Louis, MO, USA), installed on a PowerIndigo2 R8000 workstation (Silicon Graphics, Mountain View, CA, USA). Sequence alignment of the human lysosomal neuraminidase and Salmonella typhimurium neuraminidase was performed for definition of the structurally conserved regions of both target and template proteins. A fragment derived from the homologue was used to model the backbone of each structurally conserved region, and the rule-based procedure was used to generate the side chain (Sutcliffe et al. 1987).

Loop regions were then constructed by selecting a fragment for modeling loop regions from the general protein database (Jones and Thirup 1986; Claessens et al. 1989). The force field for simulation of proteins (Weiner et al. 1984, 1986) was employed in the minimization steps to optimize the geometry of a molecule. The maximum number of iterations to be performed during the minimization was set as 1000 . The minimization procedure was performed to optimize the side chain rotamers, with the backbone and the conserved side chains fixed, and then to optimize the conformation of loops, with the structurally conserved regions fixed. The mutant model was built in the same way as for the wild-type, but based on the primary structure with the amino acid replacement. To determine the influence of the replacement on the model structure, the mutant model was superimposed on the wild-type model by the least-square-mean fitting method (McLachlan 1979). We defined that the structure was influenced by the amino acid replacement when the position of an atom of the mutant differed from that of the wild-type by more than the total root-mean-square distance value.

\section{Results}

\section{Gene analysis}

The PCR products of the entire cDNA coding sequence for lysosomal neuraminidase were subcloned and sequenced. 
Fig. 1A,B. Direct sequencing of genomic DNA. The results show that both patients are heterozygous for $\mathbf{A}$ the ${ }^{649} \mathrm{G}$-to-A and $\mathbf{B}$ the ${ }^{727} \mathrm{G}$-to-A substitutions. The mutation sites are indicated by arrows. $P 1$, Patient $1 ; P 2$, patient $2 ; N$, a normal subject

A.
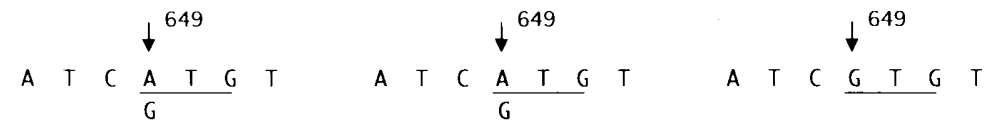

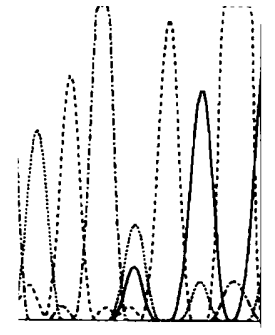

P1

B.
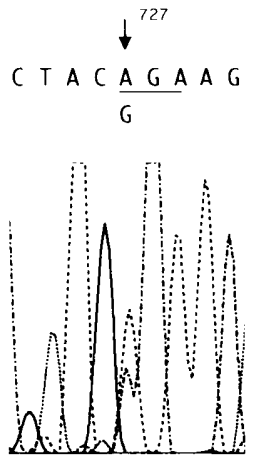

P1

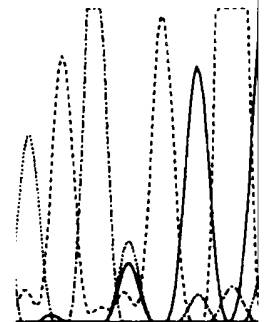

P2
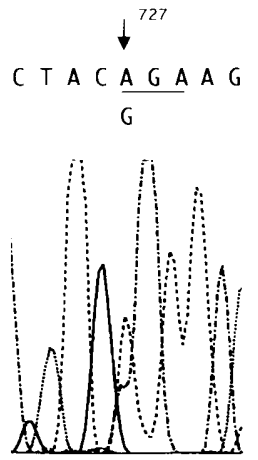

P2

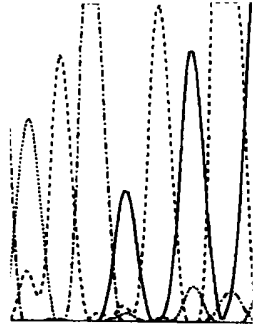

N
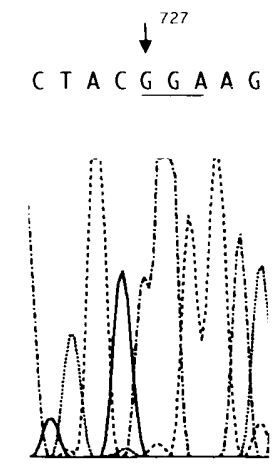

N

Table 1. Transient expression of lysosomal neuraminidase cDNA

\begin{tabular}{|c|c|c|c|c|}
\hline Plasmid & $\begin{array}{l}\text { Neuraminidase } \\
\text { (nmol/h per mg protein) }\end{array}$ & $\begin{array}{l}\text { Cathepsin A } \\
(\mu \mathrm{mol} / \mathrm{h} \text { per mg protein) }\end{array}$ & $\begin{array}{l}\beta \text {-Galactosidase } \\
\text { (nmol/h per mg protein) }\end{array}$ & $\begin{array}{l}\beta \text {-Hexosaminidase } \\
(\mu \mathrm{mol} / \mathrm{h} \text { per mg protein })\end{array}$ \\
\hline None & $<1$ & $<0.1$ & 12 & 2.2 \\
\hline apCAGGS-PP & 28 & 5.4 & 43 & 1.6 \\
\hline${ }^{\mathrm{b}} \mathrm{pCMV}$-sialidase and pCAGGS-PP & 183 & 3.7 & 39 & 1.5 \\
\hline $\begin{array}{l}\text { "pCMV-sialidase [V217M] and } \\
\text { pCAGGS-PP }\end{array}$ & 55 & 3.1 & 36 & 1.2 \\
\hline $\begin{array}{l}\text { pCMV-sialidase }[\mathrm{G} 243 \mathrm{R}] \text { and } \\
\text { pCAGGS-PP }\end{array}$ & 27 & 5.0 & 45 & 1.6 \\
\hline
\end{tabular}

Lysosomal enzyme activities in a galactosialidosis fibroblast cell line (ASVGS-1 cells) transfected with plasmids, described under "Methods". Values are the means of two independent measurements

a pCAGGS-PP, a pCAGGS vector including PPCA cDNA

bCMV-sialidase and pCAGGS-PP, a pCMV vector including the wild-type lysosomal neuraminidase cDNA and a pCAGGS vector including PPCA cDNA

${ }^{c}$ pCMV-sialidase[V217M] and pCAGGS-PP, a pCMV vector including mutant lysosomal neuraminidase cDNA with V217M and a pCAGGS vector including PPCA cDNA

${ }^{\mathrm{d}} \mathrm{pCMV}$-sialidase[G243R] and pCAGGS-PP, a pCMV vector including mutant neuraminidase cDNA with G243M and a pCAGGS vector including PPCA cDNA

Two types of cDNA clones were detected in both patients, the first having a ${ }^{649} \mathrm{G}$-to-A substitution and the other, a ${ }^{727}$ G-to-A transition (data not shown). Because both changes were located in exon 4 , the genomic DNA fragment including exon 4 was amplified and directly se- quenced. The results showed that both patients were heterozygous for the ${ }^{649} \mathrm{G}$-to-A and ${ }^{727} \mathrm{G}$-to-A substitutions (Fig. 1). The results of the cDNA and genomic DNA analyses show that both patients have one allele carrying a G-toA transition at nucleotide number 649 , resulting in the 


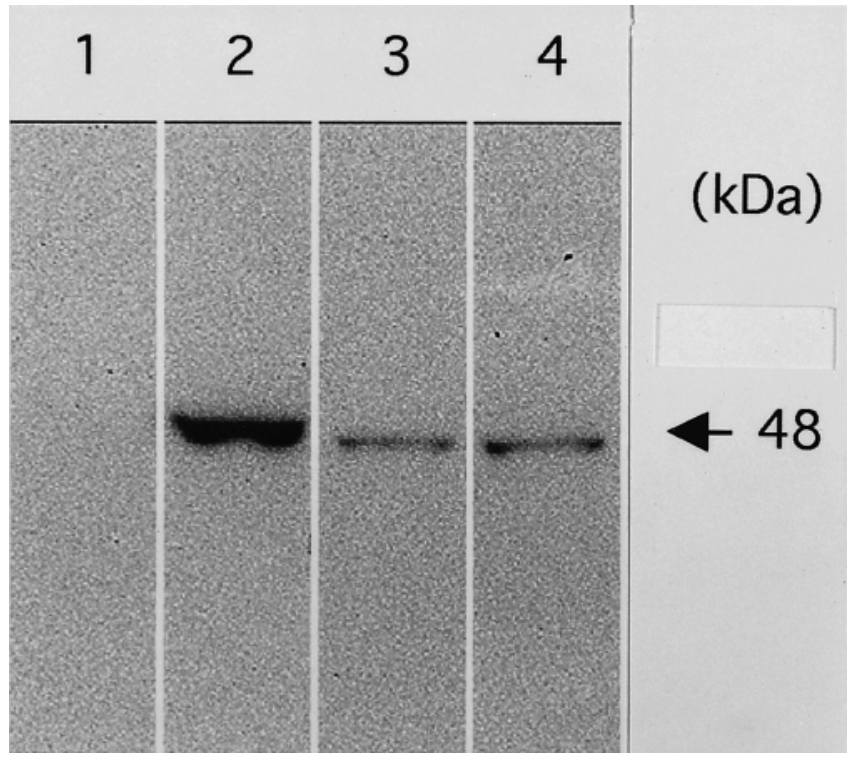

Fig. 2. Immunoblot analysis of lysosomal neuraminidase expressed in COS- 1 cells. COS-1 cells were transfected with the wild-type lysosomal neuraminidase cDNA (lane 2), mutant neuraminidase cDNA with a V217M mutation (lane 3), and that with a G243R mutation (lane 4). Non-transfected cells were used as a control (lane 1). The cell extracts were subjected to sodium dodecylsulfate (SDS) polyacrylamide gel electrophoresis (PAGE) under reducing conditions, and then immunoblotted with an anti-neuraminidase antibody, as described under "Methods" amino acid substitution of methionine for valine (V217M), and the other allele bearing a G-to-A transition at nucleotide number 727 , resulting in the substitution of arginine for glycine (G243R).

Transient expression of lysosomal neuraminidase cDNA

To characterize the effect of the amino acid substitutions, V217M and G243R, on lysosomal neuraminidase activity, the wild-type and mutant lysosomal neuraminidase cDNAs were transiently co-expressed with the human PPCA cDNA in a galactosialidosis-derived fibroblast cell line (ASVGS-1 cells). Table 1 summarizes the results. In ASVGS-1 cells, neuraminidase activity, as well as cathepsin $A$ and $\beta$-galactosidase activities, were markedly decreased by a genetic defect of PPCA, which is essential for the expression of lysosomal neuraminidase activity. Mock transfection with the PPCA cDNA alone restored the neuraminidase, $\beta$-galactosidase, and cathepsin $\mathrm{A}$ activities in the cells. Co-transfection of the wild-type lysosomal neuraminidase cDNA and PPCA cDNA caused a further seven-fold increase in neuraminidase activity compared with that in the mock-transfected cells, whereas the activity of $\beta$-hexosaminidase, as a control enzyme, did not change significantly. Co-transfection of the mutant lysosomal neuraminidase cDNA containing the ${ }^{727} \mathrm{G}$-to-A substitution (G243R) and PPCA cDNA did not cause any increase in neuraminidase activity. In contrast, a moderate increase in the enzyme activity was observed when the mutant neuraminidase cDNA containing the ${ }^{649} \mathrm{G}$-to-A substitution
Fig. 3A-D. Immunocytochemical analysis of lysosomal neuraminidase expressed transformed galactosialidosis cell lines. A Mock transfected with protective protein cathepsin A (PPCA) cDNA; B co-transfected with the wild-type lysosomal neuraminidase cDNA and PPCA cDNA; $\mathbf{C}$ co-transfected with the neuraminidase cDNA containing a Y217M mutation and PPCA cDNA; D co-transfected with the neuraminidase cDNA containing a G243R mutation and PPCA cDNA. $\times 400$
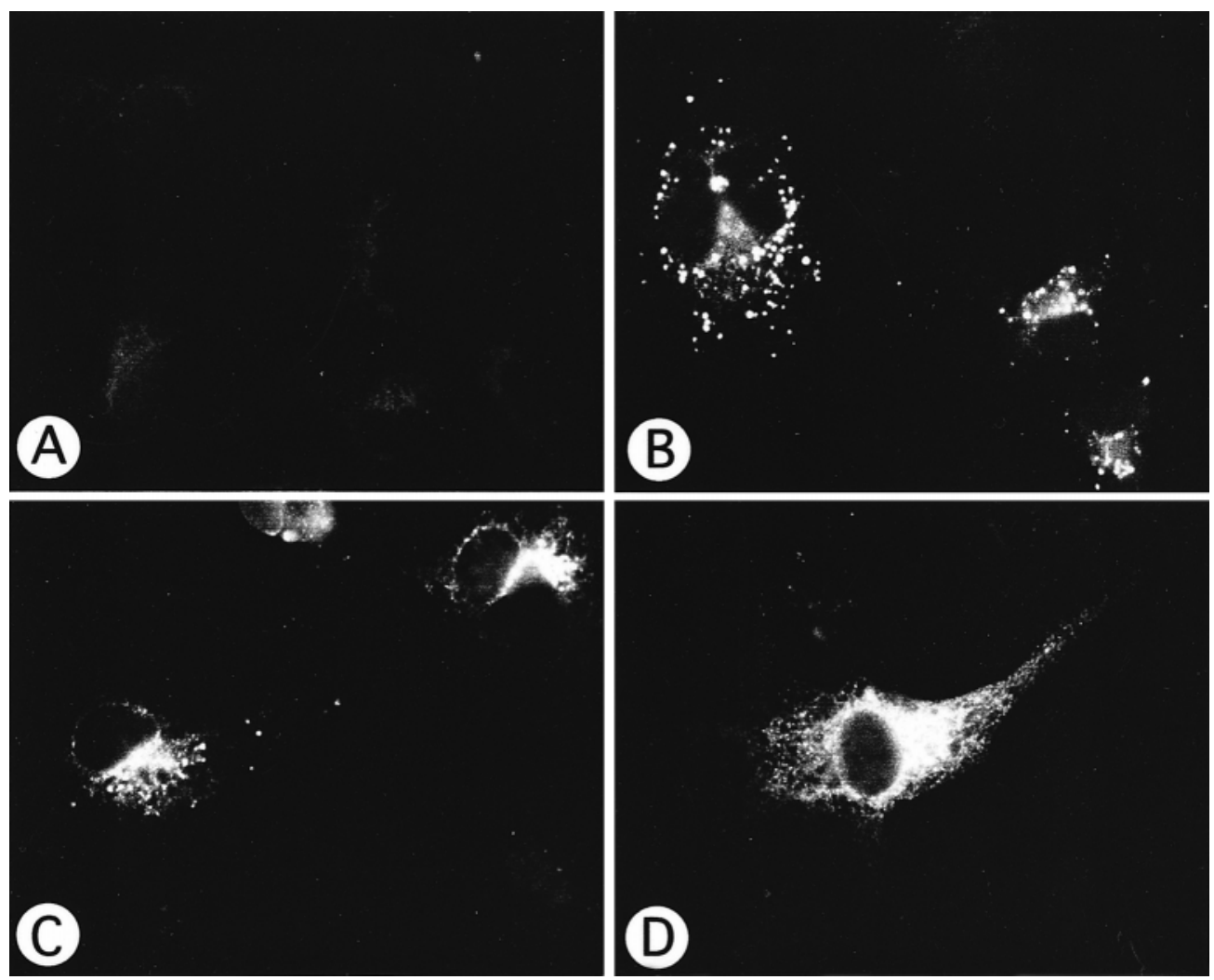
(V217M) was transfected with PPCA cDNA. In this case, neuraminidase activity was found to be restored to $17 \%$ of that in the cells co-transfected with the wild-type lysosomal neuraminidase cDNA and PPCA cDNA.

\section{Immunoblot analysis}

As shown in Fig. 2, the wild-type and mutant lysosomal neuraminidase cDNA products expressed in COS-1 cells were analyzed by immunoblotting. We used a polyclonal antibody against a recombinant human lysosomal neuraminidase fragment produced by a bacterial gene expression system that has been reported previously (Vinogradova et al. 1998). For COS-1 cells transfected with the wild-type lysosomal neuraminidase cDNA, a single 48$\mathrm{kDa}$ band was observed (Fig. 2, lane 2). The cells transfected with the mutant V217M and G243R cDNAs produced a gene product of the same size as those transfected with the wild-type cDNA (Fig. 2, lanes 3 and 4), although the intensity of the band was moderately decreased in both these cases. No other immunoreactive bands were observed.

\section{Immunocytochemical analysis}

The results of immunocytochemical analysis with the antineuraminidase antibody are shown in Fig. 3. Fluorescence microscopy revealed typical lysosomal granular staining in the cells co-transfected with the wild-type lysosomal neuraminidase cDNA and PPCA cDNA (Fig. 3B). In contrast, the expression product of lysosomal neuraminidase cDNA containing a G243R mutation showed a reticular staining pattern (Fig. 3D). On the other hand, both perinuclear reticular and partial punctate cytoplasmic staining were observed in the expression of the mutant with Y217M (Fig. 3C).

Structural modeling of wild-type and mutant lysosomal neuraminidases

According to the sequence alignment (Milner et al. 1997), we built a model of the human lysosomal neuraminidase without the N-terminal 47 residues, corresponding to the signal peptide (Milner et al. 1997; Vinogradova et al. 1998). The model was, thus, composed of the 368 amino acids from residue 48 to the terminal residue 415 , the amino acid sequence homology with the aligned fragment of Salmonella typhimurium LT2 being 39.7\%. The modeled lysosomal neuraminidase retains a basic structure composed of six four-stranded antiparallel $\beta$-sheets, and resembles a sixbladed propeller with the predicted active site cleft in the middle (Fig. 4). Both V217M and G243R are located in the $\beta$-sheet of the third $\beta$-sheet-loop unit (Fig. 4). The V217M transversion was deduced to alter the arrangement of 11 amino acid residues around the mutation site (Fig. 5). As to the G243R transversion, it was predicted to cause a drastic structural change with a wide range of up to 38 residues
(Fig. 6). Neither mutation affected the residues involved in the predicted active site (R78, R97, D103, D135, R280, and R347) (Pshezhetsky et al. 1997; Crennell et al. 1993).

\section{Discussion}

Few reports on human lysosomal neuraminidase have been published, because the enzyme has low stability during purification and requires PPCA for the expression of its activity (Verheijen et al. 1982, 1985, 1987; Hiraiwa et al. 1988; van der Horst et al. 1989). Recent cDNA cloning for human lysosomal neuraminidase has revealed that it encodes a 45$\mathrm{kDa}$ protein with three potential N-linked glycosylation sites (Bonten et al. 1996; Pshezhetsky et al. 1997; Milner et al. 1997). This information has facilitated the identification of gene mutations causing sialidosis. So far, only one nonsense mutation, E377X, has been found in a patient with sialidosis type 1 (Bonten et al. 1996). In sialidosis type 2, three missense mutations, L91R (Bonten et al. 1996), F260Y (Pshezhetsky et al. 1997), and L363P (Pshezhetsky et al. 1997); a four-base duplication causing a frameshift (Pshezhetsky et al. 1997); and a single-base deletion, resulting in a frameshift and extending the protein by 69 amino acid residues (Bonten et al. 1996), have been reported.

In this study, we identified two novel gene mutations in two Japanese patients with sialidosis type 1. Although these patients are unrelated according to our investigations, they are both compound heterozygotes for the ${ }^{649} \mathrm{G}$-to-A and ${ }^{727} \mathrm{G}$-to-A alterations. The base substitutions are found at the $\mathrm{CpG}$ site, which is known as a mutation hot spot (Cooper and Youssoufian 1988).

Using the information regarding the tertiary structure of bacterial neuraminidase, we modeled the structure of human lysosomal neuraminidase, and deduced the structural changes caused by the identified mutations. Both the V217M and G243R mutations were distant from the deduced active residues. The simulation suggests that the G243R replacement can cause a drastic widespread change in the protein structure. The biochemical study showed that the G243R mutant expressed low amounts of a protein with the same molecular size as in that in the wild type, but it could not be transported to the lysosomal compartment. The enzyme activity of the expressed product with the G243R mutation was completely abolished. The structural change is likely to cause a folding defect, which results in a transport defect and intracellular degradation and/or a defect of activation. On the other hand, the deduced structural change caused by $\mathrm{V} 217 \mathrm{M}$ is smaller than that caused by G243R. The V217M mutant was partly transported to lysosomes, and showed residual enzyme activity.

Both patients described here are compound heterozygotes for V217M and G243M. Considering the results of this study, we surmise that the V217M mutation causes a mild change in the protein structure compatible with the presence of residual enzyme activity. Therefore, this mutation may be closely related to the phenotype with a late onset and moderate clinical course. 
Fig. 4. Structural model of lysosomal neuraminidase and the locations of the mutations identified in two Japanese sialidosis patients. The backbone is colored according to secondary structures; $\alpha$-helix (red), $\beta$ strand (blue), and coil (yellow). The sequence motifs conserved in the sialidase family, five Asp-boxes (Ser/Thr-X-Asp-X-Gly-X-X-Trp/Phe), a Phe-Arg-Ile-Pro sequence motif, and a C-terminal, Tyr-X-X-hydrophobic residue motif are colored cyan, orange, and white, respectively. The side chains of the predicted active residues are colored magenta. The residues of V217 and G243, where the amino acid residue replacements will occur in the patients, are colored green

Fig. 5. Conformational change in lysosomal neuraminidase caused by V217M mutation. The amino acid residues influenced by the change from V217 (green) to M217 (red) are shown; those colored blue are the wild-type residues and those colored yellow are the mutant residues. The active site residues are colored magenta
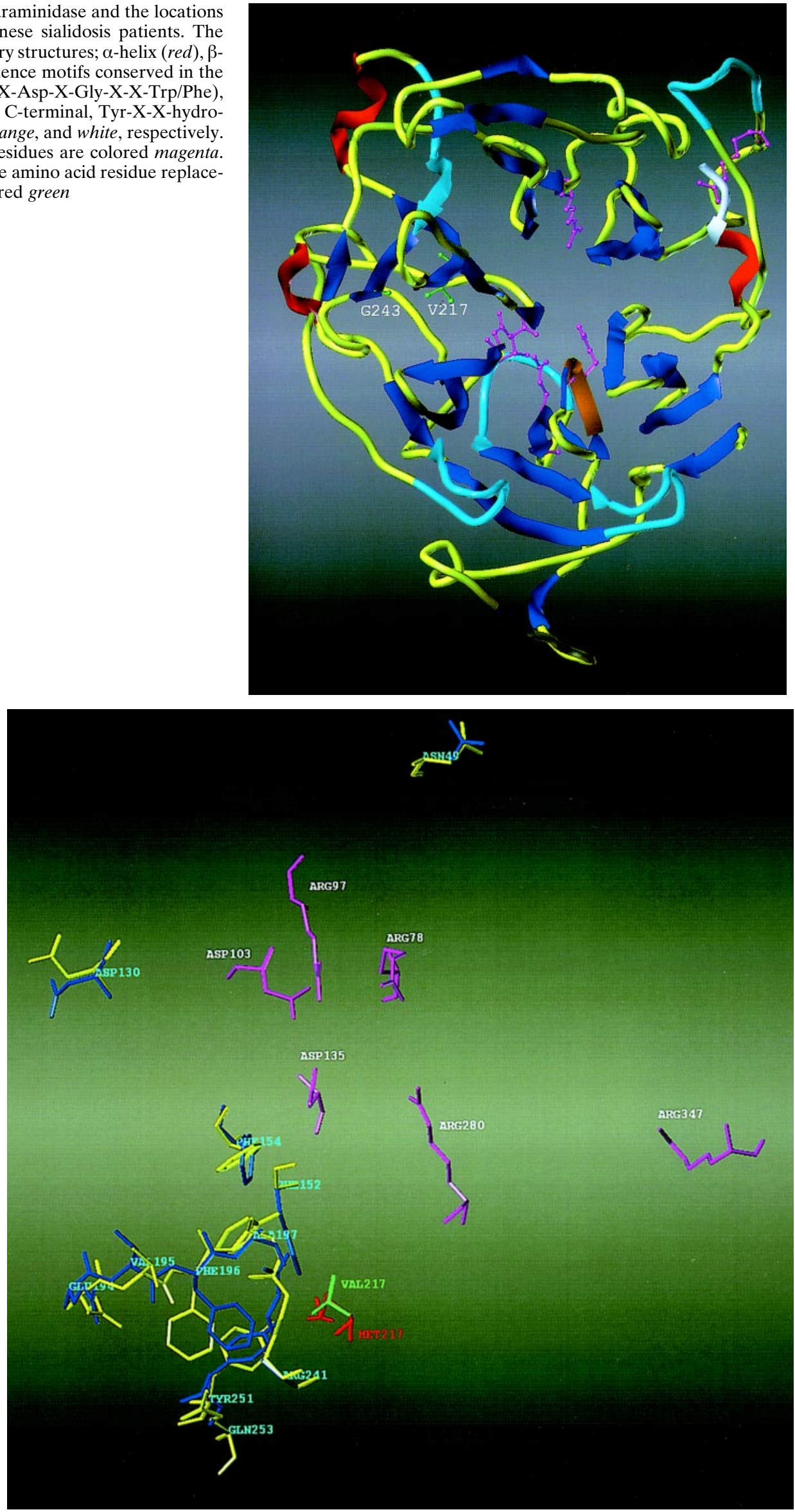
Fig. 6. Conformational change in lysosomal neuraminidase caused by G243R mutation. The amino acid residues influenced by the change from G243 (green) to R243 (red) are shown; those colored blue are the wild-type residues and those colored yellow are the mutant residues. The active residues are colored magenta

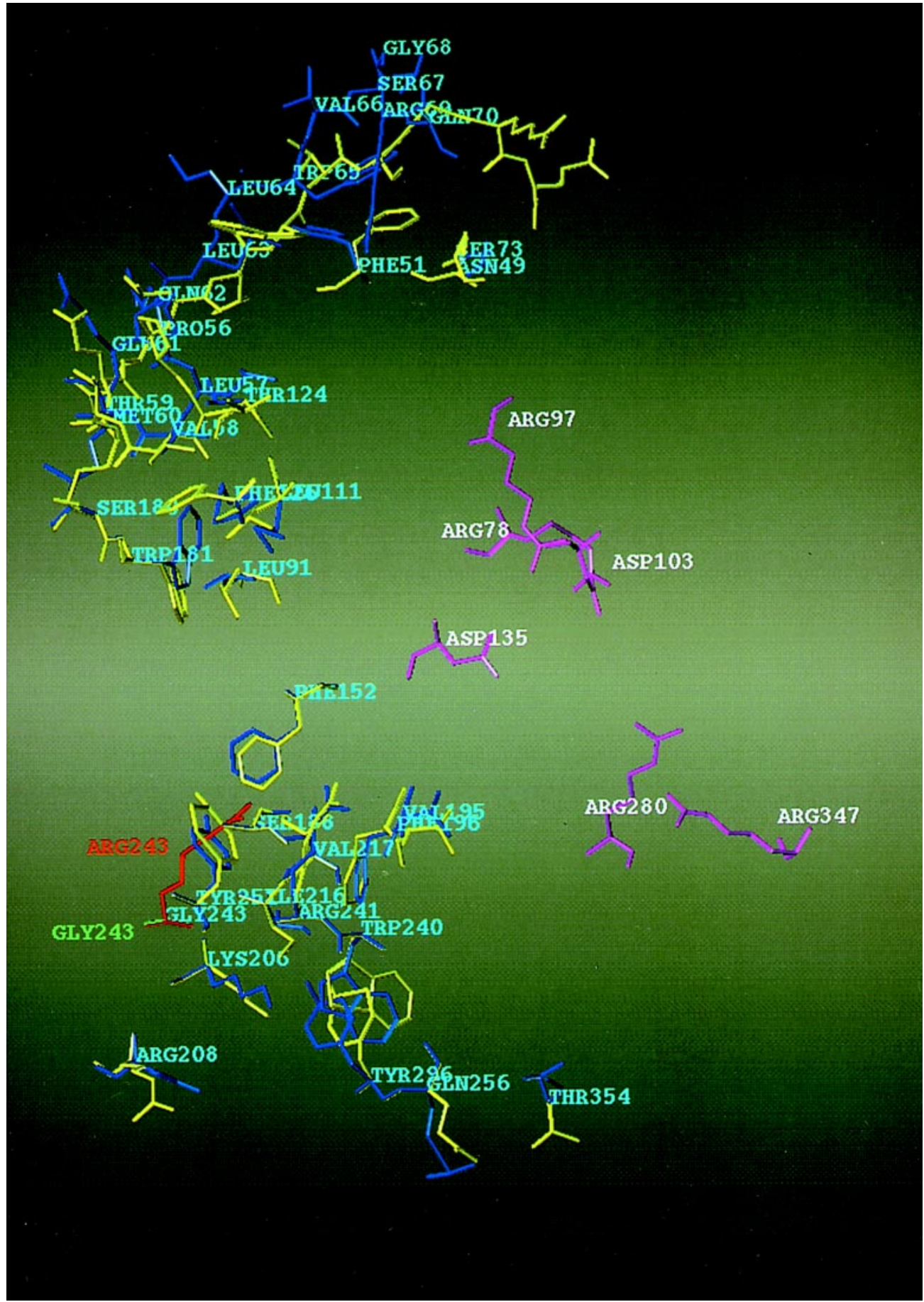

In conclusion, we identified two novel gene mutations in Japanese patients with sialidosis type 1 , and characterized the structural and functional defects caused by these missense mutations. Molecular and structural studies will facilitate clarification of the complicated pathophysiology of sialidosis.

Acknowledgments We thank Dr. Michel Potier for helpful advice.

This work was partly supported by grants from the Tokyo Metropolitan Government, the Ministry of Education, Science, Sports and Culture of Japan, and the Ministry of Health and Welfare of Japan.

\section{References}

Bonten E, van der Spoel A, Fornerod M, Grosveld G, d'Azzo A (1996) Characterization of human lysosomal neuraminidase defines the molecular basis of the metabolic storage disorder sialidosis. Genes Dev 10:3156-3169

Claessens M, van Cutsem E, Lasters I, Wodak S (1989) Modeling the polypeptide backbone with "spare parts" from known protein structures. Protein Eng 2:335-345

Cooper DN, Youssoufian H (1988) The CpG dinucleotide and human genetic disease. Hum Genet 78:151-155 
Crennell SJ, Garman EF, Laver WG, Vimr ER, Taylor GL (1993) Crystal structure of a bacterial sialidase (from Salmonella typhimurium LT2) shows the same fold as an influenza virus neuraminidase. Proc Natl Acad Sci USA 90:9852-9856

d'Azzo A, Hoogeveen A, Reuser AJ, Robinson D, Galjaard H (1982) Molecular defect in combined beta-galactosidase and neuraminidase deficiency in man. Proc Natl Acad Sci USA 79:4535-4539

d'Azzo A, Andria G, Strisciuglio P, Galjaard H (1995) Galactosialidosis. In: Scriver CR, Beaudet AL, Sly WS, Valle D (eds) The metabolic and molecular bases of inherited disease, 7th edn. McGraw-Hill, New York, pp 2825-2837

Davis LG, Dibner MD, Battey JF (1986) Basic methods in molecular biology. Elsevier, New York

Gluzman Y (1981) SV40-transformed simian cells support the replication of early SV40 mutants. Cell 23:175-182

Hiraiwa M, Nishizawa M, Uda Y, Nakajima T, Miyatake T (1988) Human placental sialidase: further purification and characterization. J Biochem 103:86-90

Itoh K, Takiyama N, Nagao Y, Oshima A, Sakuraba H, Potier M, Suzuki Y (1991) Acid carboxypeptidase deficiency in galactosialidosis. Jpn J Hum Genet 36:171-178

Jones TA, Thirup S (1986) Using known substructures in protein model building and crystallography. EMBO J 5:819-822

Kawasaki ES (1990) Amplification of RNA. In: Innis MA, Gelfand DH, Sninsky JJ, White TJ (eds) PCR protocols. A guide to methods and applications. Academic Press, San Diego, pp 21-27

Laemmli UK (1970) Cleavage of structure proteins during the assembly of the head bacteriophage T4. Nature 227:680-685

MacGregor GR, Caskey CT (1989) Construction of plasmids that express $E$. coli $\beta$-galactosidase in mammalian cells. Nucleic Acids Res 17:2365

McLachlan AD (1979) Gene duplications in the structural evolution of chymotrypsin. J Mol Biol 128:49-79

Milner CM, Smith SV, Carrillo MB, Taylor GL, Hollinshead M, Campbell RD (1997) Identification of a sialidase encoded in the human major histocompatibility complex. J Biol Chem 272:4549-4558

Mitoma H, Uchiyama T, Yokota T, Furukawa T, Tsukagoshi H (1993) Action myoclonus induced guided movement. J Neurol 241:92-95

Miyazaki J, Takaki S, Araki K, Tashiro F, Tominaga A, Takatsu K, Yamamura K (1991) Efficient selection for high-expression transfectants with a novel eukaryotic vector. Gene 108:193-200

Pshezhetsky AV, Richard C, Michaud L, Igdloura S, Wang S, Elsliger M-A, Qu J, Leclerc D, Dallaire L, Potier M (1997) Cloning, expression and chromosomal mapping of human lysosomal sialidase and characterization of mutations in sialidosis. Nature Genet 15:316320

Sakuraba H, Itoh K, Shimmoto M, Utsumi K, Kase R, Hashimoto Y, Ozawa T, Ohwada Y, Imataka G, Eguchi M, Furukawa T, Schepers U, Sandhoff K (1999) GM2 gangliosidosis AB variant. Clinical and biochemical studies of a Japanese patient. Neurology 52:372-377

Shimmoto M, Sakuraba H, Suzuki Y (1993) Protective protein gene mutations in galactosialidosis. J Clin Invest 91:2393-2398
Sussman JL, Lin D, Jiang J, Manning NO, Prilusky J, Ritter O, Abola EE (1998) Protein data bank (PDB): database of three-dimensional structural information of biological macromolecules. Acta Crystallogr D54:1078-1084

Sutcliffe MJ, Hayes FRF, Blundell TL (1987) Knowledge based modeling of homologous proteins, part II: rules for the conformations of substituted side chains. Protein Eng 1:385-392

Suzuki K (1987) Enzymatic diagnosis of sphingolipidoses. In: Ginsbury $\mathrm{V}(\mathrm{ed})$ Methods in enzymology, vol 138. Academic Press, New York, pp 727-762

Suzuki Y, Sakuraba H, Potier M, Akagi M, Sakai M, Beppu H (1981) $\beta$-Galactosidase-neuraminidase deficiency in adults: deficiency of a freeze-labile neuraminidase in leukocytes and fibroblasts. Hum Genet 58:387-389

Thomas GH, Beaudet AL (1995) Disorders of glycoprotein degradation and structure: $\alpha$-mannosidosis, $\beta$-mannosidosis, fucosidosis, sialidosis, aspartylglucosaminuria, and carbohydrate-deficient glycoprotein syndrome. In: Scriver CR, Beaudet AL, Sly WS, Valle D (eds) The metabolic and molecular bases of inherited disease, 7th edn. McGraw-Hill, New York, pp 2529-2561

van der Horst G, Galjart A, d'Azzo A, Galjaard H, Verheijen FW (1989) Indentification and in vitro reconstitution of lysosomal neuraminidase from human placenta. J Biol Chem 264:1317-1322

van der Spoel A, Bonten E, d'Azzo A (1998) Transport of human lysosomal neuraminidase to mature lysosomes requires protective protein/cathepsin A. EMBO J 17:1588-1597

Verheijen FW, Brossmer R, Galjaard H (1982) Purification of acid $\beta$ galactosidase and acid neuraminidase from bovine testis: evidence for an enzyme complex. Biochem Biophys Res Commun 108:868875

Verheijen FW, Palmeri S, Hoogeveen AT, Galjaard H (1985) Human placental neuraminidase. Activation, stabilization and association with $\beta$-galactosidase and its protective protein. Eur $\mathbf{J}$ Biochem 149:315-321

Verheijen FW, Palmeri S, Galjaard H (1987) Purification and partial characterization of lysosomal neuraminidase from human placenta. Eur J Biochem 162:63-67

Vinogradova MV, Michaudo L, Mezentsev AV, Lukong KE, El-Alfy M, Morales CR, Potier M, Pshezhetsky AV (1998) Molecular mechanism of lysosomal sialidase deficiency in galactosialidosis involves its rapid degradation. Biochem J 330:641-650

Weiner SJ, Kollman PA, Case DA, Singh UC, Ghio C, Alagona G, Profeta S Jr, Weiner P (1984) A new force field for molecular mechanical simulation of nucleic acids and proteins. J Am Chem Soc 106:765-784

Weiner SJ, Kollman PA, Nguyen DT, Case DA (1986) An all atom force field for simulations of proteins and nucleic acids. J Comput Chem 7:230-252

Yamamoto M, Yamauchi T, Yamamoto K, Kobayashi T (1995) Activated sialidase activity in transformed lymphocytes by Epstein-Barr (EB) virus of sialidosis type I (cherry-red spot-myoclonus syndrome). J Neurol Sci 131:105-107 